\title{
Random partial orders defined by angular domains
}

\author{
Paul Balister* $\quad$ Balázs Patkós ${ }^{\dagger}$
}

July 19, 2010

\begin{abstract}
The $d$-dimensional random partial order is the intersection of $d$ independently and uniformly chosen (with replacement) linear orders on the set $[n]=$ $\{1,2, \ldots, n\}$. This is equivalent to picking $n$ points uniformly at random in the $d$-dimensional unit cube $Q_{d}=[0,1]^{d}$ with the coordinate-wise ordering. If $d=2$, then this can be rephrased by declaring that for any pair $P_{1}, P_{2} \in Q_{2}$ we have $P_{1} \prec P_{2}$ if and only if $P_{2}$ lies in the positive upper quadrant defined by the two axis-parallel lines crossing at $P_{1}$. In this paper we study the random partial order with parameter $\alpha(0 \leq \alpha \leq \pi)$ which is generated by picking $n$ points uniformly at random from $Q_{2}$ equipped with the same partial order as above but with the quadrant replaced by an angular domain of angle $\alpha$.
\end{abstract}

\section{Introduction}

There exists several models that generate random partial orders on $n$ elements. A very good, but not very recent survey is given in [9]. One of the most studied models (introduced by Winkler $[16,17]$ ) is to consider the intersection of $d$ randomly and independently chosen linear orders on the same $n$ element ground set. This is called the $d$-dimensional random partial order. One of the nice features of this model is that it has the same distribution as $n$ uniformly chosen points in the $d$-dimensional unit cube $Q_{d}=[0,1]^{d}$ with respect to coordinate-wise ordering. Sometimes it is convenient to consider the model in which points of $Q_{d}$ are generated according to a Poisson process of intensity $n$. Standard techniques (see e.g., $[3,4,9]$ ) show that the 'same' results hold in both models for all problems studied so far, and therefore

\footnotetext{
*Department of Mathematics, University of Memphis, Memphis, TN 38152, USA. Partially supported by NSF Grant CCF-0728928. E-mail: pbalistr@memphis.edu

$\dagger$ Department of Computer Science, University of Memphis, Memphis, TN 38152, USA. Supported by NSF Grant CCF-0728928. E-mail: bpatkos@memphis.edu
} 
for convenience we shall often prove results for one model and use it to deduce the corresponding result for the other. The problem that attracted the most attention of researchers is finding the largest chain or antichain (respectively a set of pairwise comparable or incomparable elements). This problem was already raised by Ulam [14] in the case $d=2$ where the question is equivalent to that of finding the longest increasing subsequence of a random permutation. The right order of magnitude was determined by Hammersley [11], and the asymptotically precise answer was given by Logan and Schepp [13] and Vershik and Kerov [15]. Their result states that the size $L_{n}$ of the largest chain is concentrated around its mean, which is $(2+o(1)) \sqrt{n}$. Research on how sharp the concentration was initialized by Frieze [10], Bollobás and Brightwell [3] and Bollobás and Johansson [5]. An upper bound on the variance of $L_{n}$ that later turned out to be tight was established by Kim [12] and Baik, Deift and Johansson proved the matching lower bound, moreover they determined the limiting distribution of $\frac{L_{n}-2 \sqrt{n}}{n^{1 / 6}}$. A survey on the method of their proof (together with some applications) is given by Aldous and Diaconis in [1].

The problem was partially solved for general $d$ by Bollobás and Winkler [7].

In this paper, we consider a generalization of the $d=2$ case. Note that in the coordinate-wise ordering for any 2 points $P_{1}, P_{2}$ in the plane we have $P_{1} \prec P_{2}$ if and only if $P_{2}$ lies in the positive upper quadrant defined by the two axis-parallel lines crossing at $P_{1}$. Modifying the angle $\frac{\pi}{2}$ to any angle $\alpha, 0 \leq \alpha \leq \pi$, we obtain a partial order that we shall call the $\alpha$-ordering. More precisely, for any pair of points $P_{1}=\left(x_{1}, y_{1}\right)$ and $P_{2}=\left(x_{2}, y_{2}\right)$ in $\mathbb{R}^{2}$ we define $P_{1} \prec_{\alpha} P_{2}$ if and only if $x_{1}+y_{1}<x_{2}+y_{2}$ and $P_{2}$ lies in the open angular domain at $P_{1}$ of angle $\alpha$ with angle bisector parallel to the line $x=y$. The probability space of the partial order obtained by selecting $n$ points uniformly at random from $Q:=Q_{2}=[0,1]^{2}$ and considering the partial suborder of the $\alpha$-ordering induced by these points will be denoted by $\mathcal{P}_{\alpha, n}$ and the probability space obtained by points generated by a Poisson process of intensity $n$ in $Q$ will be denoted by $\mathcal{P}_{\alpha, n}^{P}$. Note that with probability 1 both $\mathcal{P}_{\alpha, n}$ and $\mathcal{P}_{\alpha, n}^{P}$ form an antichain if $\alpha=0$ and a chain (i.e., a linear or total ordering) if $\alpha=\pi$. The corresponding comparability graphs (in which every vertex corresponds to an element of the partial order and two vertices are joined by an edge if the corresponding elements are comparable in the partial order) will be denoted by $G_{\alpha, n}$ and $G_{\alpha, n}^{P}$, and we will use the notation $L_{\alpha, n}$ and $L_{\alpha, n}^{P}$ for the lengths of the longest chains in $\mathcal{P}_{\alpha, n}$ or $\mathcal{P}_{\alpha, n}^{P}$.

As we will show in Section 2, a quite natural transformation yields that $\mathcal{P}_{\alpha, n}$ is equivalent to picking $n$ points randomly in some rhombus and consider them equipped with the original $\frac{\pi}{2}$-ordering. This will enable us to determine $L_{\alpha, n}$ for most values of $\alpha$. In view of this transformation, one might question whether it is interesting enough to study these angle-parametrized models at all. Our main reason to believe so is that letting $\alpha$ range from 0 to $\pi$, our random partial order evolves from an antichain 
to a chain and the comparability graph evolves from an empty graph to a complete graph. In this way, we can study "hitting time" problems and compare the results to other random processes (like the Erdös-Rényi graphs process [8]). More precisely, if $\mathfrak{P}$ is a monotone poset property (e.g. containing a chain of size at least $k$, having a connected comparability graph, having a comparability graph of diameter at most $l$, etc.), then one can define

$$
\left.\tau(\mathfrak{P})=\lim _{\varepsilon \rightarrow 0^{+}} \liminf _{n \rightarrow \infty} \inf \left\{\alpha: \mathbb{P}\left(\mathcal{P}_{\alpha, n} \in \mathfrak{P}\right) \leq 1-\varepsilon\right)\right\} .
$$

We will prove that $\tau(\mathfrak{P})=\frac{\pi}{2}$ when $\mathfrak{P}$ is the property of having a connected comparability graph.

The rest of the paper is organized as follows: Section 2 contains the above mentioned transformation and results on $L_{\alpha, n}$. In particular we prove the following theorem.

Theorem 1.1. Let $\alpha=\alpha_{n}$ satisfy

$$
\omega\left((\log n)^{2} n^{-1}\right) \leq \alpha \leq \pi-\omega\left(n^{-1}\right) .
$$

Then both $L_{\alpha, n} / \sqrt{n \tan \frac{\alpha}{2}}$ and $L_{\alpha, n}^{P} / \sqrt{n \tan \frac{\alpha}{2}}$ tend to 2 in probability as $n \rightarrow \infty$.

As a converse to Theorem 1.1 we shall also show that this result fails for both $L_{\alpha, n}$ and $L_{\alpha, n}^{P}$ when $\alpha=o\left((\log n)^{2} n^{-1}\right)$ or $\alpha=\pi-o\left(n^{-1}\right)$.

Section 3 addresses the problem of determining the diameter of $G_{\alpha, n}$ when $\alpha>\frac{\pi}{2}$. Winkler [17] settled the case $\alpha=\frac{\pi}{2}$ even for general dimension $d$ showing that the diameter is 3 with probability tending to 1 as $n$ tends to infinity (with high probability, w.h.p.). Our main result is the following theorem.

Theorem 1.2. The following holds with high probability

$$
\operatorname{diam}\left(G_{\alpha, n}\right)=\left\{\begin{array}{l}
1 \quad \text { if } \pi-\alpha=o\left(n^{-2}\right) \\
2 \quad \text { if } \pi-\alpha=\omega\left(n^{-2}\right) \text { and } \alpha-\frac{\pi}{2}=\omega\left(n^{-1 / 2}\right) \\
3 \quad \text { if } 0 \leq \alpha-\frac{\pi}{2}=o\left(n^{-1 / 2}\right) .
\end{array}\right.
$$

As $G_{\alpha, n}^{P}=G_{\alpha, X}$ where $X$ is a Poisson variable of mean $n$, and since $X=(1+o(1)) n$ w.h.p., corresponding results also hold for $G_{\alpha, n}^{P}$.

In Section 3 we also study the probability of the event that $G_{\alpha, n}$ is connected for any $\alpha<\frac{\pi}{2}$. In particular, we determine the expected number of connected components of $G_{\alpha, n}$. Section 4 contains some concluding remarks and open problems. 
Notation. Throughout the paper $Q$ denotes the unit square $[0,1]^{2}$. Given a partial ordering on $Q$ and a point $P \in Q$ we shall write $P^{+}=\left\{P^{\prime} \in Q: P \prec P^{\prime}\right\}$ and $P^{-}=\left\{P^{\prime} \in Q: P^{\prime} \prec P\right\}$ for the set of elements larger, respectively smaller than $P$. We shall also write $P^{\|}=P^{+} \cup P^{-} \cup\{P\}$ and $P^{\perp}=Q \backslash P^{\|}$for the set of elements comparable, respectively incomparable to $P$. If $P=(x, y)$, then we will use the notation $P^{+}(x, y), P^{-}(x, y), P^{\|}(x, y), P^{\perp}(x, y)$. For a set $S$ of elements we write $S^{\|}=$ $\bigcap_{P \in S} P^{\|}$. For a point $P \in Q$, the lines forming $\partial P^{\perp}$ in the $\alpha$-ordering $\prec_{\alpha}$ will be denoted by $e_{\alpha,+, P}$ and $e_{\alpha,-, P}$.

\section{Longest chains, antichains}

In this section, we address the problem of finding $L_{\alpha, n}$ for $\alpha \in(0, \pi)$. Note that if we denote by $T$ the rotation by angle $\frac{\pi}{2}$ around the point $\left(\frac{1}{2}, \frac{1}{2}\right)$, then for any $P_{1}, P_{2} \in Q$ we have that $P_{1}$ and $P_{2}$ are comparable in the $\alpha$-ordering if and only if $T P_{1}$ and $T P_{2}$ are incomparable in the $(\pi-\alpha)$-ordering. Thus if $W_{\alpha, n}$ denotes the size of the largest antichain in $\mathcal{P}_{\alpha, n}$, then $L_{\alpha, n}$ and $W_{\pi-\alpha, n}$ have the same distribution and hence all results about $L_{\alpha, n}$ or $L_{\alpha, n}^{P}$ can be converted to results about $W_{\pi-\alpha, n}$ or $W_{\pi-\alpha, n}^{P}$.

Let us begin with stating the theorem concerning the size of the longest chain in $\mathcal{P}_{\frac{\pi}{2}, n}$ or $\mathcal{P}_{\frac{\pi}{2}, n}^{P}$

Theorem 2.1 (Logan, Shepp [13]; Vershik, Kerov [15]). Both $L_{\frac{\pi}{2}, n} / \sqrt{n}$ and $L_{\frac{\pi}{2}, n}^{P} / \sqrt{n}$ tend to 2 in probability as $n \rightarrow \infty$.

In our proofs, we will use the following concentration result:

Theorem 2.2 (Kim [12]). If $0<\theta \leq n^{1 / 3} / 20$, then

$$
\left.\mathbb{P}\left(\mid L_{\frac{\pi}{2}, n}^{P}-2 \sqrt{n}\right) \mid>\theta n^{1 / 6}\right) \leq \exp \left(-\frac{4 \theta^{3 / 2}}{3}+\phi(\theta)\right),
$$

where

$$
\phi(\theta):=\left(\frac{\theta}{27 n^{1 / 3}}+\frac{5 \log n}{\theta^{1 / 2} n^{1 / 3}}\right) \theta^{3 / 2} .
$$

Let $R_{\alpha}$ denote the rhombus with two of its vertices being $(0,0),(1,1)$ and having angle $\alpha$ at these vertices. Note that the area of $R_{\alpha}$ is $\tan \frac{\alpha}{2}$. Furthermore, for any measurable subset $S \subseteq \mathbb{R}^{2}$, write $\mathcal{P}_{\alpha, n}^{P}(S)$ for the probability space of random partial orders obtained by placing points in $\mathbb{R}^{2}$ according to a Poisson process of intensity $n$ and then taking the $\alpha$-ordering of those points of this process that lie in $S$. Thus $\mathcal{P}_{\alpha, n}^{P}=\mathcal{P}_{\alpha, n}^{P}(Q)=\mathcal{P}_{\alpha, n}^{P}\left(R_{\frac{\pi}{2}}\right)$. We shall often vary the set $S$, in which case we consider the Poisson process on $\mathbb{R}^{2}$ as being fixed and each $\mathcal{P}_{\alpha, n}^{P}(S)$ a partial suborder of the 
same partial order $\mathcal{P}_{\alpha, n}^{P}\left(\mathbb{R}^{2}\right)$. Let $T^{\alpha}$ denote the linear transformation of the plane that fixes the line $y=x$ and takes $(1,0)$ to the vertex of $R_{\alpha}$ lying in the half-plane $x>y$ and $(0,1)$ to the vertex of $R_{\alpha}$ lying in the half-plane $x<y$. Thus the image of $Q$ under $T^{\alpha}$ is just $R_{\alpha}$. Clearly, for any $P_{1}, P_{2} \in \mathbb{R}^{2}$ we have $P_{1} \prec_{\frac{\pi}{2}} P_{2}$ if and only if $T^{\alpha} P_{1} \prec{ }_{\alpha} T^{\alpha} P_{2}$. Thus, we obtain the following proposition.

Proposition 2.3. The probability spaces $\mathcal{P}_{\frac{\pi}{2}, n}^{P}$ and $\mathcal{P}_{\alpha, n / \tan \frac{\alpha}{2}}^{P}\left(R_{\alpha}\right)$ have the same distribution.

The linear transformation $\left(T^{\alpha}\right)^{-1}$ takes the unit square to the rhombus $R_{\beta}$ with the property that $\tan \frac{\beta}{2}=\left|R_{\beta}\right|=\left|R_{\alpha}\right|^{-1}=\left(\tan \frac{\alpha}{2}\right)^{-1}$. Thus $\beta=\pi-\alpha$. Therefore by applying $\left(T^{\alpha}\right)^{-1}=T^{\pi-\alpha}$ we obtain another proposition that we will use in our proofs.

Proposition 2.4. The probability spaces $\mathcal{P}_{\alpha, n}^{P}$ and $\mathcal{P}_{\frac{\pi}{2}, n \tan \frac{\alpha}{2}}^{P}\left(R_{\pi-\alpha}\right)$ have the same distribution.

Proof of Theorem 1.1. We first prove the result for $L_{\alpha, n}^{P}$ by splitting the argument into two cases.

CASE I: $\alpha \geq \frac{\pi}{2}$ and $\alpha \leq \pi-\omega\left(n^{-1}\right)$.

By Proposition 2.4, $L_{\alpha, n}^{P}$ has the same distribution as $L_{\frac{\pi}{2}, n \tan \frac{\alpha}{2}}^{P}\left(R_{\pi-\alpha}\right)$. As $\alpha \geq \frac{\pi}{2}$ we have $R_{\pi-\alpha} \subseteq Q$, so $L_{\frac{\pi}{2}, n \tan \frac{\alpha}{2}}^{P}\left(R_{\pi-\alpha}\right) \leq L_{\frac{\pi}{2}, n \tan \frac{\alpha}{2}}^{P}$, which by Theorem 2.1 is at most $(2+\varepsilon) \sqrt{n \tan \frac{\alpha}{2}}$ w.h.p. as $n \tan \frac{\alpha}{2} \geq n \rightarrow \infty$. For the lower bound, let $S_{0}$ be the largest square centered at $\left(\frac{1}{2}, \frac{1}{2}\right)$ that lies in $R_{\pi-\alpha}$. Inductively define $S_{i}$ for $i>0$ so that $S_{i+1}$ is the largest square in $R_{\pi-\alpha}$ centered at a point on the line $y=x$ whose bottom left corner is the top right corner of $S_{i}$. Similarly define $S_{-i}, i>0$, as the largest square in $R_{\pi-\alpha}$ whose top right corner is the bottom left corner of $S_{-i+1}$. Clearly any two points $P \in S_{i}, P^{\prime} \in S_{j}, i \neq j$, are comparable in the $\frac{\pi}{2}$-ordering. Thus one can obtain a chain in $\mathcal{P}_{\frac{\pi}{2}}^{P}, n \tan \frac{\alpha}{2}\left(R_{\pi-\alpha}\right)$ by taking the union of the largest chains in each $\mathcal{P}_{\frac{\pi}{2}, n \tan \frac{\alpha}{2}}^{P}\left(S_{i}\right)$. Let the side length of $S_{i}$ be $s_{i}$ and choose $\ell$ minimal so that $\sum_{i=-\ell}^{\ell} s_{i} \geq 1-2 \varepsilon$. Then for $|i| \leq \ell$, the point in $S_{i}$ closest to $\left(\frac{1}{2}, \frac{1}{2}\right)$ is at least $\varepsilon$ from the boundary of $Q$ and so $s_{i} \geq \varepsilon\left(1-\tan \left(\frac{\alpha}{2}-\frac{\pi}{4}\right)\right)=\Theta(\varepsilon(\pi-\alpha))$. Thus the expected number of points in each $S_{i}$ is $s_{i}^{2} n \tan \frac{\alpha}{2}=\Omega\left(\varepsilon^{2} n(\pi-\alpha)\right)=\omega(1)$ for any fixed $\varepsilon>0$. Thus we can apply Theorem 2.1 and deduce that for $n$ sufficiently large, each $S_{i}$ contains a chain of length at least $(2-\varepsilon) s_{i} \sqrt{n \tan \frac{\alpha}{2}}$ with probability at least $1-\varepsilon^{2}$, say. If $I$ is the set of $i$ such that $S_{i}$ does not contain such a chain, then $\mathbb{E}\left(\sum_{i \in I} s_{i}\right) \leq \varepsilon^{2} \sum_{i=-\ell}^{\ell} s_{i} \leq \varepsilon^{2}$. Thus with probability at least $1-\varepsilon, \sum_{i \in I} s_{i} \leq \varepsilon$ and $\mathcal{P}_{\frac{\pi}{2}, n \tan \frac{\alpha}{2}}^{P}\left(R_{\pi-\alpha}\right)$ contains a chain of length at least

$$
\sum_{i \notin I}(2-\varepsilon) s_{i} \sqrt{n \tan \frac{\alpha}{2}} \geq(2-\varepsilon)((1-2 \varepsilon)-\varepsilon) \sqrt{n \tan \frac{\alpha}{2}} \geq(2-7 \varepsilon) \sqrt{n \tan \frac{\alpha}{2}}
$$


as required.

CASE II: $\alpha \leq \frac{\pi}{2}$ and $\alpha \geq \omega\left((\log n)^{2} / n\right)$.

By Proposition 2.4 we can work in $\mathcal{P}_{\frac{\pi}{2}, n \tan \frac{\alpha}{2}}^{P}\left(R_{\pi-\alpha}\right)$. As $Q \subseteq R_{\pi-\alpha}$ we obtain $L_{\alpha, n}^{P} \geq(2-\varepsilon) \sqrt{n \tan \frac{\alpha}{2}}$ w.h.p. by Theorem 2.1. To prove an upper bound on $L_{\alpha, n}^{P}$ let us consider a pair of points $P_{1}, P_{2}$ in $R_{\pi-\alpha}$ generated by the Poisson process. If these points are not comparable, then they cannot be contained in a chain. Otherwise, the length $L$ of the largest chain having these points as endpoints is distributed as $2+L_{\frac{\pi}{2}}^{P}, s n \tan \frac{\alpha}{2}(Q)$, where $s$ is the area of the axis-parallel rectangle $S$ determined by $P_{1}$ and $P_{2}$. Indeed, $S$ is the set of points that lie between $P_{1}$ and $P_{2}$ in the coordinate-wise ordering. Furthermore, for any axis-parallel rectangle $S$ the distribution of $L_{\frac{\pi}{2}, m}^{P}(S)$ depends only on the area of $S$ (and is independent of the ratio of the length of the sides). Observe that the largest axis-parallel rectangle in $R_{\pi-\alpha}$ is the unit square $Q$ and thus $s \leq 1$. By embedding $S$ in a larger rectangle if necessary, we can stochastically bound each $L_{\frac{\pi}{2}, m}^{P}(S)$ by $L_{\frac{\pi}{2}, m}^{P}(Q)$. Thus by Theorem 2.2 , we obtain that for a fixed pair $P_{1}, P_{2}$ the probability that there is a chain larger than $(2+\varepsilon) \sqrt{n \tan \frac{\alpha}{2}}$ is not more than $e^{-\frac{4}{3} \theta^{3 / 2}+\phi(\theta)}$ where we may take $\theta=\left(n \tan \frac{\alpha}{2}\right)^{1 / 3} / 20$. But then $\theta=\omega\left((\log n)^{2 / 3}\right)$ by the assumption on $\alpha$. Hence $e^{-\lambda^{2}} e^{-\frac{4}{3} \theta^{3 / 2}+\phi(\theta)}=o\left(1 / n^{2}\right)$. The probability that the number of points generated by the Poisson process is greater than $2 n$ (and thus the number of pairs is greater than $2 n^{2}$ ) is $\exp (-\Omega(n)$ ), thus $L_{\alpha, n}^{P} \leq(2+\varepsilon) \sqrt{n \tan \frac{\alpha}{2}}+2$ w.h.p..

Finally by choosing Poisson variables $X$ and $Y$ of means $(1-\varepsilon) n$ and $(1+\varepsilon) n$ respectively, we have that for any $\varepsilon>0, X \leq n \leq Y$ holds w.h.p.. Thus as $L_{\alpha, n}$ is monotonic in $n, L_{\alpha,(1-\varepsilon) n}^{P}=L_{\alpha, X} \leq L_{\alpha, n} \leq L_{\alpha, Y}=L_{\alpha,(1+\varepsilon) n}^{P}$ w.h.p., and so the result also follows for $L_{\alpha, n}$.

We note that if $\alpha=\pi-o\left(n^{-1}\right)$ then $\sqrt{n \tan \frac{\alpha}{2}}=\omega(n)$. As there are only $n$ Poisson points on average in $Q$, the conclusion of Theorem 1.1 cannot possibly hold in this case. The next theorem shows that it also fails to hold when $\alpha=o\left((\log n)^{2} / n\right)$.

Theorem 2.5. Assume $\alpha=o\left((\log n)^{2} / n\right)$. Then

$$
\mathbb{P}\left(L_{\alpha, n}^{P} \geq 3 \sqrt{n \tan \frac{\alpha}{2}}\right) \rightarrow 1
$$

as $n \rightarrow \infty$.

Proof. By Proposition 2.4 we can work in $\mathcal{P}_{\frac{\pi}{2}, n \tan \frac{\alpha}{2}}^{P}\left(R_{\pi-\alpha}\right)$. Let $s=\sqrt{n \tan \frac{\alpha}{2}}$ and tile $\mathbb{R}^{2}$ with squares $S_{i, j}=(i / 4 s, j / 4 s)+[0,1 / 4 s]^{2}$ of side length $1 / 4 s$. For each $i$, let $\mathcal{C}_{i}=\left\{S_{i+j, j}: S_{i+j, j} \subseteq R_{\pi-\alpha}\right\}$ be the set of these squares in a diagonal sequence that lie inside $R_{\pi-\alpha}$. Then there exists a $K>0$ so that $\mathcal{C}_{i}$ consists of at least $3 s$ squares for all $i$ with $|i| \leq K \alpha^{-1}$. If each of the squares in $\mathcal{C}_{i}$ contains a point of the Poisson 
process, then one obtains a chain of length $3 s$. The expected number of points in one of these squares is $1 / 16$, so this happens with probability $\left(1-e^{-1 / 16}\right)^{-3 s} \geq e^{-9 s}$. The events that each square in $\mathcal{C}_{i}$ contain a point are independent for distinct $i$. Thus if $K \alpha^{-1} e^{-9 s} \rightarrow \infty$ then w.h.p. a chain of length $3 s$ will exist. However this occurs as $s=\Theta\left((n \alpha)^{1 / 2}\right)=o(\log n)$ and $\alpha^{-1}=\omega\left(n^{1 / 2}\right)$.

By comparing $L_{\alpha, n}^{P}$ with $L_{\alpha, n}$ we deduce that if $\alpha=o\left((\log n)^{2} / n\right)$ then $\mathbb{P}\left(L_{\alpha, n} \geq\right.$ $\left.(3-\varepsilon) \sqrt{n \tan \frac{\alpha}{2}}\right) \rightarrow 1$.

\section{Connectivity}

Winkler [16] showed that $G_{\frac{\pi}{2}, n}$ is connected and has diameter 3 w.h.p.. In this section we consider the case when $\alpha \neq \frac{\pi}{2}$. After some easy preliminary results, we address the case of $\alpha$ larger than $\frac{\pi}{2}$ and prove Theorem 1.2 in several parts. The following proposition is straightforward but will be useful in the proofs of our results.

Proposition 3.1. The area $\left|P^{\|}(x, y)\right|$ is maximized at $x=y=0$ with

$$
\left|P^{\|}(0,0)\right|=\min \left\{1,1-\tan \left(\frac{\pi}{4}-\frac{\alpha}{2}\right)\right\} .
$$

Furthermore, if $P(x, y) \in Q \backslash\left(P^{\|}(0,0) \cup P^{\|}(1,1)\right)$ and $x \leq y$, then

$$
\left|P^{\|}(x, y)\right|=\left(x^{2}+(1-y)^{2}\right)\left(\tan \left(\frac{\pi}{4}+\frac{\alpha}{2}\right)-\tan \left(\frac{\pi}{4}-\frac{\alpha}{2}\right)\right) / 2=\left(x^{2}+(1-y)^{2}\right) \tan \alpha .
$$

Theorem 3.2. Each of the following occur with high probability,

(i) $\mathcal{P}_{\alpha, n}$ contains a pair of incomparable elements if $\alpha=\pi-\omega\left(n^{-2}\right)$,

(ii) $\mathcal{P}_{\alpha, n}$ is a linear ordering (so the diameter of $G_{\alpha, n}$ is 1) if $\alpha=\pi-o\left(n^{-2}\right)$,

(iii) $\mathcal{P}_{\alpha, n}$ contains a pair of comparable elements if $\alpha=\omega\left(n^{-2}\right)$,

(iv) $\mathcal{P}_{\alpha, n}$ is an antichain if $\alpha=o\left(n^{-2}\right)$.

Proof. We prove (iii) and (iv), then (i) and (ii) follow by applying the coupling between $\mathcal{P}_{\alpha, n}$ and $\mathcal{P}_{\pi-\alpha, n}$ defined by the rotation by angle $\frac{\pi}{2}$ around the point $\left(\frac{1}{2}, \frac{1}{2}\right)$.

W.l.o.g. we may assume that $\alpha=o(1)$. Let $X_{i, j}$ denote the event that $P_{i}, P_{j} \in$ $\mathcal{P}_{\alpha, n}$ are comparable and let us write $X=\sum_{1 \leq i<j \leq n} X_{i, j}$ for the number of pairs of comparable elements. Clearly, we have

$$
\mathbb{E}(X)=\left(\begin{array}{l}
n \\
2
\end{array}\right) \mathbb{E}\left(X_{i, j}\right)=\left(\begin{array}{l}
n \\
2
\end{array}\right) \int_{0}^{1} \int_{0}^{1}\left|P^{\|}(x, y)\right| d x d y
$$


By Proposition 3.1, $\left|P^{\|}(x, y)\right|=\left(x^{2}+(1-y)^{2}\right) \tan \alpha$ except on a set of size at most $2\left|P^{\|}(0,0)\right|$ where it is at most $\left|P^{\|}(0,0)\right|=\Theta(\tan \alpha)$. Thus

$$
\mathbb{E}\left(X_{i, j}\right)=2 \iint_{0 \leq x<y \leq 1}\left(x^{2}+(1-y)^{2}\right) \tan \alpha d x d y+O\left(\left|P^{\|}(0,0)\right|^{2}\right)=\Theta(\alpha) .
$$

Hence by the assumption $\alpha=o(1)$ we conclude that $\mathbb{E}(X)=\Theta\left(n^{2} \alpha\right)$. This gives that if $\alpha=o\left(n^{-2}\right)$, then $\mathbb{E}(X)=o(1)$, which proves (iv), while if $\alpha=\omega\left(n^{-2}\right)$ then $\mathbb{E}(X) \rightarrow \infty$. Note that if $\{i, j\} \cap\left\{i^{\prime}, j^{\prime}\right\}=\emptyset$ then the random variables $X_{i, j}$ and $X_{i^{\prime}, j^{\prime}}$ are independent. Furthermore, as Proposition 3.1 states $\left|P^{\|}(x, y)\right|$ is maximized at $\left|P^{\|}(0,0)\right|$, we have

$$
\mathbb{E}\left(X_{i, j} X_{i, k}\right)=\mathbb{P}\left(X_{i, j}=1\right) \mathbb{E}\left(X_{i, k} \mid X_{i, j}=1\right) \leq \mathbb{E}\left(X_{i, j}\right)\left|P^{\|}(0,0)\right| .
$$

Therefore

$$
\begin{aligned}
\operatorname{Var}(X) & =\sum_{i, j, i^{\prime}, j^{\prime}}\left(\mathbb{E}\left(X_{i, j} X_{i^{\prime}, j^{\prime}}\right)-\mathbb{E}\left(X_{i, j}\right) \mathbb{E}\left(X_{i^{\prime}, j^{\prime}}\right)\right) \\
& \leq n\left(\begin{array}{c}
n-1 \\
2
\end{array}\right) \mathbb{E}\left(X_{i, j}\right)\left|P^{\|}(0,0)\right|+\left(\begin{array}{c}
n \\
2
\end{array}\right) \mathbb{E}\left(X_{i, j}\right) \\
& =O\left(n^{3} \alpha^{2}+n^{2} \alpha\right) .
\end{aligned}
$$

Thus $\operatorname{Var}(X)=o\left((\mathbb{E} X)^{2}\right)$ and (iii) follows from Chebyshev's inequality.

Theorem 3.3. If $\alpha=\frac{\pi}{2}+\omega\left(n^{-1 / 2}\right)$, then the diameter of $G_{\alpha, n}$ is at most 2 .

Proof. For any $0<x \leq \frac{1}{2}$ let $Q_{x}^{-}=[0, x]^{2}$ and $Q_{x}^{+}=[1-x, 1]^{2}$. Note that for any $P \in Q \backslash Q_{1 / 2}^{+}$we have $Q_{x}^{+} \subseteq P^{+}$if $\left(\frac{1}{2}-x\right) \tan \frac{\alpha-\pi / 2}{2} \geq x$ and similarly for any $P \in Q \backslash Q_{1 / 2}^{-}$we have $Q_{x}^{-} \subseteq P^{-}$if $\left(\frac{1}{2}-x\right) \tan \frac{\alpha-\pi / 2}{2} \geq x$. By the assumption on $\alpha$ we obtain that this inequality holds for some $x=\omega\left(n^{-1 / 2}\right)$ which implies that both $Q_{x}^{+}$ and $Q_{x}^{-}$contain a point from $\mathcal{P}_{\alpha, n}$ w.h.p..

By these observations, if $P_{1}, P_{2} \in Q \backslash Q_{1 / 2}^{+}$or $P_{1}, P_{2} \in Q \backslash Q_{1 / 2}^{-}$, then they have a common neighbor in $G_{\alpha, n}$ w.h.p., and if neither of the above hold, then $P_{1} \in Q_{1 / 2}^{+}$, $P_{2} \in Q_{1 / 2}^{-}$, and thus $P_{2} \prec P_{1}$ or $P_{2} \in Q_{1 / 2}^{+}, P_{1} \in Q_{1 / 2}^{-}$and thus $P_{1} \prec P_{2}$.

Theorem 3.2 (i) and Theorem 3.3 yield the following corollary.

Corollary 3.4. If both $\alpha-\frac{\pi}{2}=\omega\left(n^{-1 / 2}\right)$ and $\pi-\alpha=\omega\left(n^{-2}\right)$ hold, then the diameter of $G_{\alpha, n}$ is 2 w.h.p..

The following theorem states that the lower bound of Theorem 3.3 is tight. Its proof is basically the same as Winkler's proof in [17] but to simplify the independency argument we work with the Poisson model. 


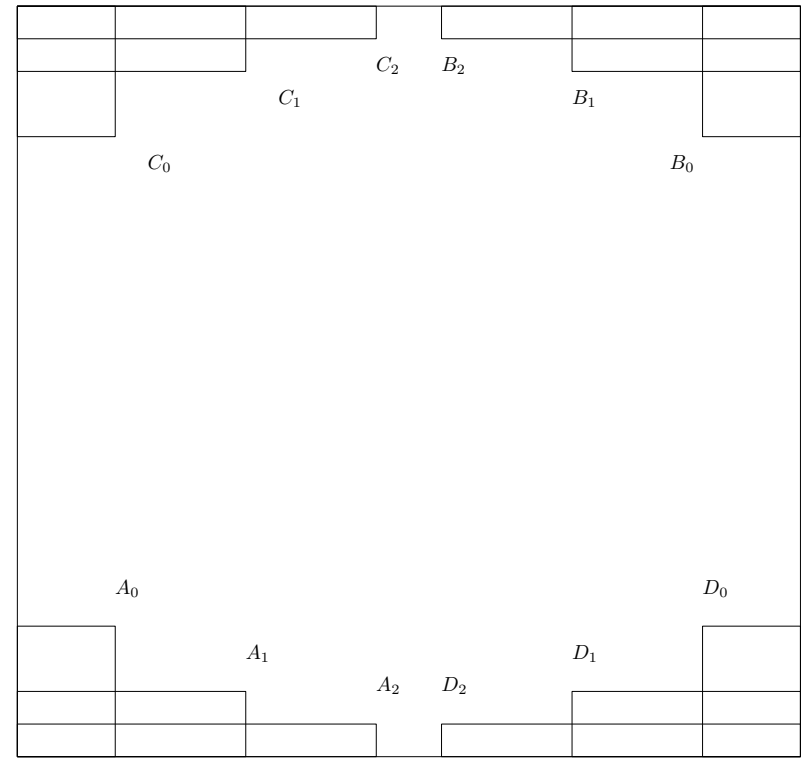

Figure 1: Finding points at distance 3.

Theorem 3.5. Let $\alpha=\frac{\pi}{2}+o\left(n^{-1 / 2}\right)$. Then w.h.p. the diameter of $G_{\alpha, n}^{P}$ is 3 .

Proof. The fact that the diameter is at most 3 w.h.p. comes from Winkler's result that the diameter of $G_{\frac{\pi}{2}, n}$ (and hence also $G_{\frac{\pi}{2}, n}^{P}$ ) is 3 w.h.p.. Hence it is enough to show that w.h.p. there exist $P_{1}, P_{2} \in \mathcal{P}_{\alpha, n}^{P}$ with $P_{1}^{\|} \cap P_{2}^{\|} \cap \mathcal{P}_{\alpha, n}^{P}=\emptyset$.

Write $\alpha=\frac{\pi}{2}+\frac{1}{\beta n^{1 / 2}}$ where $\beta=\beta_{n} \rightarrow \infty$. Fix an integer $\ell$ and construct rectangles $A_{0}, A_{1}, \ldots, A_{\ell}, B_{0}, B_{1}, \ldots, B_{\ell}, C_{0}, C_{1}, \ldots, C_{\ell}, D_{0}, D_{1}, \ldots, D_{\ell}$ as in Figure 1. Rectangles $A_{i}, B_{i}, C_{i}, D_{i}$ will each have width $\beta^{\frac{i}{\ell}} n^{-1 / 2}$ and height $\beta^{-\frac{i}{\ell}} n^{-1 / 2}$, hence their area is $1 / n$ and the probability that they contain a point in $\mathcal{P}_{\alpha, n}^{P}$ is $1-1 / e$.

Let $J \subseteq Q$ denote the set of points that are contained in at least two of the rectangles defined above and let $A_{i}^{\prime}=A_{i} \backslash J, B_{i}^{\prime}=B_{i} \backslash J, C_{i}^{\prime}=C_{i} \backslash J, D_{i}^{\prime}=$ $D_{i} \backslash J$. The area of the region $A_{i} \cap \bigcup_{j>i} A_{j}$ is $\beta_{n}^{-1 / \ell} n^{-1}$, so the area of $J$ is at most $4 \ell \beta^{-1 / \ell} n^{-1}=o\left(n^{-1}\right)$. Thus $J \cap \mathcal{P}_{\alpha, n}^{P}=\emptyset$ w.h.p.. Note that for sufficiently large $n$, $\tan \left(\frac{\alpha}{2}-\frac{\pi}{4}\right) \leq \frac{1}{\beta n^{1 / 2}}$ and $\beta^{1 / \ell}>2$, so $\tan \left(\frac{\alpha}{2}-\frac{\pi}{4}\right) \leq \beta^{-(i-1) / \ell} n^{-1 / 2}-\beta^{-i / \ell} n^{-1 / 2}$ for all $i \leq \ell$. Therefore if say $P=(x, y) \in C_{i}^{\prime}$ and $P^{\prime}=\left(x^{\prime}, y^{\prime}\right) \in B_{j}^{\prime}$ with $P \prec_{\alpha} P^{\prime}$, then $y^{\prime}>y-\beta_{n}^{-(i-1) / \ell} n^{-1 / 2}+\beta_{n}^{-i / \ell} n^{-1 / 2}$ and so $j \geq i-1$. Similarly, if $P \in D_{i}^{\prime}$ and $P^{\prime} \in B_{j}^{\prime}$ with $P \prec_{\alpha} P^{\prime}$ then $j \leq i+1$. Corresponding results hold for $P^{\prime} \in A_{j}^{\prime}$, so for any pair of points $P_{1} \in C_{i}^{\prime}, P_{2} \in D_{i}^{\prime}$ we have $\left(P_{1}^{\|} \cap P_{2}^{\|}\right) \backslash J \subseteq A_{i-1}^{\prime} \cup A_{i}^{\prime} \cup A_{i+1}^{\prime} \cup B_{i-1}^{\prime} \cup$ $B_{i}^{\prime} \cup B_{i+1}^{\prime}$. Let $E_{j}$ denote the event that $C_{3 i+1}^{\prime} \cap \mathcal{P}_{\alpha, n}^{P}$ and $D_{3 i+1}^{\prime} \cap \mathcal{P}_{\alpha, n}^{P}$ are not empty but $\left(A_{i-1}^{\prime} \cup A_{i}^{\prime} \cup A_{i+1}^{\prime} \cup B_{i-1}^{\prime} \cup B_{i}^{\prime} \cup B_{i+1}^{\prime}\right) \cap \mathcal{P}_{\alpha, n}^{P}$ is empty. By the above, if $n$ is large 
enough then any of the events $E_{0}, E_{1}, \ldots, E_{(\ell-1) / 3}$ would provide us a pair of points whose distance in $G_{\alpha, n}$ is at least 3 . As these events are independent, the probability that none of these events hold is at least $\left(1-e^{-6}\left(1-e^{-2}\right)^{2}\right)^{\ell / 3}$ for large $n$, which is smaller than any fixed $\varepsilon>0$ provided $\ell>\ell(\varepsilon)$.

Corollary 3.6. Let $\alpha=\frac{\pi}{2}+o\left(n^{-1 / 2}\right)$. Then w.h.p. the diameter of $G_{\alpha, n}$ is 3 .

Proof. The fact that the diameter is at most 3 w.h.p. comes from Winkler's result that the diameter of $G_{\frac{\pi}{2}, n}$ is 3 w.h.p.. Hence as above it is enough to show that w.h.p. there exist $P_{1}, P_{2} \in \mathcal{P}_{\alpha, n}$ with $P_{1}^{\|} \cap P_{2}^{\|} \cap \mathcal{P}_{\alpha, n}=\emptyset$. Let $X$ be a Poisson variable with mean $n+n^{2 / 3}$. Then by Theorem 3.5, w.h.p. there exist $P_{1}, P_{2} \in \mathcal{P}_{\alpha, X}$ with $P_{1}^{\|} \cap P_{2}^{\|} \cap \mathcal{P}_{\alpha, X}=\emptyset$. But w.h.p. $X \geq n$, so by removing $X-n$ points of $\mathcal{P}_{\alpha, X}$ at random we obtain an instance of $\mathcal{P}_{\alpha, n}$. Clearly $P_{1}^{\|} \cap P_{2}^{\|} \cap \mathcal{P}_{\alpha, n}=\emptyset$, and, conditioned on $X$, with probability at most $n^{2} / X^{2}$ neither $P_{1}$ nor $P_{2}$ is removed. Since $n^{2} / X^{2}=1-O\left(n^{-1 / 3}\right)$ w.h.p., the result follows.

Now we turn to the case $\alpha<\frac{\pi}{2}$. The following definition is the key ingredient to understanding what can make $G_{\alpha, n}$ disconnected.

Definition 3.7. Let $\mathcal{P}$ be a finite partial suborder of $Q$ equipped with the $\alpha$-ordering. We say that $C \in Q$ (not necessarily in $\mathcal{P}$ !) is a cut point of $\mathcal{P}$ if $C^{\|} \cap \mathcal{P}=\emptyset$.

We will need the following lemma.

Lemma 3.8. Let $\mathcal{P}$ be a finite partial suborder of $Q$ equipped with the $\alpha$-ordering where $\alpha>0$. Then its comparability graph $G$ is disconnected if and only if at least one of the following the possibilities holds:

(i) $|\mathcal{P}|>1$ and there exists $P \in \mathcal{P}$ such that $P$ is a cut point of $\mathcal{P}$.

(ii) there exists $P_{1}, P_{2} \in \mathcal{P}$ such that the intersection $C=\left(c_{1}, c_{2}\right)$ of $e_{\alpha,+, P_{1}}$ and $e_{\alpha,-, P_{2}}$ is a cut point of $\mathcal{P}$ and there exists points $P=(x, y)$ and $P^{\prime}=\left(x^{\prime}, y^{\prime}\right)$ of $\mathcal{P}$ with $x-y<c_{1}-c_{2}<x^{\prime}-y^{\prime}$.

Proof. Case (i) corresponds to an isolated vertex in the comparability graph $G$ and a cut point satisfying (ii) defines a cut in $G$. To see the other direction of the lemma, let us suppose $G$ is disconnected. If there exists an isolated vertex in $G$, then the corresponding element of $\mathcal{P}$ is a cut point and (i) holds. If there is no isolated vertex, then consider a "leftmost" component of $G$, i.e., one which contains an element $P=(x, y)$ of $\mathcal{P}$ with $x-y$ being minimal. Note that components can be ordered by the values of $x-y$ of their points, since if $P=(x, y)$ does not lie in a component $\mathcal{C}$ of $G$ and if $\mathcal{C}$ contains points $P^{\prime}=\left(x^{\prime}, y^{\prime}\right)$ and $P^{\prime \prime}=\left(x^{\prime \prime}, y^{\prime \prime}\right)$ with 
$x^{\prime}-y^{\prime}<x-y<x^{\prime \prime}-y^{\prime \prime}$ then by considering a path from $P^{\prime}$ to $P^{\prime \prime}$ it must contain two such points that are comparable. But then either $P^{\prime}$ or $P^{\prime \prime}$ is comparable to $P$. Let $P_{1}\left(P_{2}\right)$ be the element in this component with $e_{\alpha,+, P_{1}}\left(e_{\alpha,-, P_{2}}\right)$ being closest to the point $(1,0)$. Let $C$ be the intersection of $e_{\alpha,+, P_{1}}$ and $e_{\alpha,-, P_{2}}$. Then $C$ is a cut point as if $P \in C^{+} \cap \mathcal{P}$ then $P \succ_{\alpha} P_{2}$ but $e_{\alpha,+, P}$ lies to the right of $e_{\alpha,+, P_{1}}$, and similarly if $P \in C^{-} \cap \mathcal{P}$ then $P \prec_{\alpha} P_{1}$ but $e_{\alpha,-, P}$ lies to the right of $e_{\alpha,-, P_{2}}$. If $C=P_{1}$ or $C=P_{2}$ (and in particular, if $P_{1}=P_{2}$ ) then $C$ is an isolated vertex and (i) holds. By choice of leftmost component, any point $P=(x, y)$ in this component satisfies $x-y<c_{1}-c_{2}$ and any point $P^{\prime}=\left(x^{\prime}, y^{\prime}\right)$ not in this component satisfies $c_{1}-c_{2}<x^{\prime}-y^{\prime}$. Thus (ii) holds.

Proposition 3.9. If $\alpha=o(1)$ then $G_{\alpha, n}$ is disconnected w.h.p..

Proof. We claim that $C=\left(\frac{1}{\alpha^{1 / 3} \sqrt{n}}, 1-\frac{1}{\alpha^{1 / 3} \sqrt{n}}\right)$ is a cutpoint of $G_{\alpha, n}$. Indeed, by Proposition 3.1 we have $\left|C^{\|}\right|=\frac{2}{\alpha^{2 / 3} n} \tan \alpha=O\left(\frac{\alpha^{1 / 3}}{n}\right)=o(1 / n)$ and thus $C^{\|}$is empty w.h.p., while both squares $\left[0, \frac{1}{\alpha^{1 / 3} \sqrt{n}}\right] \times\left[1-\frac{1}{\alpha^{1 / 3} \sqrt{n}}, 1\right]$ and $\left[\frac{1}{\alpha^{1 / 3} \sqrt{n}}, 1\right] \times\left[0,1-\frac{1}{\alpha^{1 / 3} \sqrt{n}}\right]$ are non-empty w.h.p.. Therefore $G_{\alpha, n}$ is disconnected.

Proposition 3.10. For any fixed $\alpha$ with $0<\alpha<\frac{\pi}{2}$ there exist constants $0<p_{\alpha}, p_{\alpha}^{\prime}<$ 1 such that

$$
p_{\alpha} \leq \mathbb{P}\left(G_{\alpha, n}^{P} \text { is connected }\right) \leq p_{\alpha}^{\prime} .
$$

Proof. The existence of $p_{\alpha}^{\prime}$ follows from the fact that the point $C=\left(\frac{1}{\sqrt{n}}, 1-\frac{1}{\sqrt{n}}\right)$ is a cut point of $G_{\alpha, n}^{P}$ with positive probability.

We still have to prove a lower bound on the probability of $G_{\alpha, n}^{P}$ being connected. Write $t=\tan \frac{\alpha}{2}$ and let $x_{1}, x_{2}, \ldots, x_{m}$ be a geometric sequence with ratio $r=1+t$, $x_{1}=\frac{1}{\sqrt{n}}$ and $x_{m}$ the largest element of the sequence with $x_{m}<1$. Define squares in the following way: for each integer $1 \leq i \leq m / 2$ let $I_{2 i-1}=\left[0, \frac{t}{2} x_{2 i-1}\right] \times[1-(1+$ $\left.\left.\frac{t}{2}\right) x_{2 i-1}, 1-x_{2 i-1}\right]$ and $I_{2 i}=\left[x_{2 i},\left(1+\frac{t}{2}\right) x_{2 i}\right] \times\left[1-\frac{t}{2} x_{2 i}, 1\right]$. Let $U_{i}$ and $V_{i}$ denote the top-left and bottom-right corner of $I_{i}$. Note that $e_{\alpha,-, V_{2 i}}$ meets the line $x=0$ at the point $P=\left(0,1-\frac{t}{2} x_{2 i}-\left(1+\frac{t}{2}\right) x_{2 i} \tan \left(\frac{\pi}{4}-\frac{\alpha}{2}\right)\right)$. Now $\tan \left(\frac{\pi}{4}-\frac{\alpha}{2}\right)=(1-t) /(1+t)$ and $x_{2 i}=(1+t) x_{2 i-1}$ so $P=\left(0,1-\frac{1}{2}(t(1+t)+(2+t)(1-t)) x_{2 i-1}\right)=\left(0,1-x_{2 i-1}\right)=U_{2 i-1}$. By symmetry, $e_{\alpha,+, U_{2 i+1}}$ meets $V_{2 i}$, so $I_{2 i-1}$ and $I_{2 i+1}$ lie between $e_{\alpha,-, U_{2 i}}$ and $e_{\alpha,+, V_{2 i}}$. Hence $I_{2 i-1}, I_{2 i+1} \prec_{\alpha} I_{2 i}$. Thus $I_{1} \prec_{\alpha} I_{2} \succ_{\alpha} I_{3} \prec_{\alpha} I_{4} \succ_{\alpha} \ldots$ Let $J_{i}$ be the image of $I_{i}$ when reflected to the line $x=y$, therefore we also have $J_{1} \prec_{\alpha} J_{2} \succ_{\alpha} J_{3} \prec_{\alpha} J_{4} \succ_{\alpha} \ldots$

Let $E_{1}$ denote the event that all $I_{i}$ s and $J_{j}$ s are non-empty, let $E_{2}$ be the event that $\mathcal{P}_{\alpha, n}^{P}$ does not contain any point in $S=\left[0, x_{2}\right] \times\left[1-x_{1}, 1\right] \cup\left[1-x_{1}, 1\right] \times\left[0, x_{2}\right]$ and let $E_{3}$ denote the event that the points of $\mathcal{P}_{\alpha, n}^{P}$ in $R_{\alpha} \cup I_{m} \cup J_{m}$ lie in the same component of $G_{\alpha, n}^{P}$. The events $E_{1}$ and $E_{2}$ are independent as they involve disjoint areas. $\mathbb{P}\left(E_{1}\right)=\prod_{i=1}^{m}\left(1-e^{-r^{2(i-1)}}\right)^{2} \geq \prod_{i=1}^{\infty}\left(1-e^{-r^{2(i-1)}}\right)^{2}>0$. 


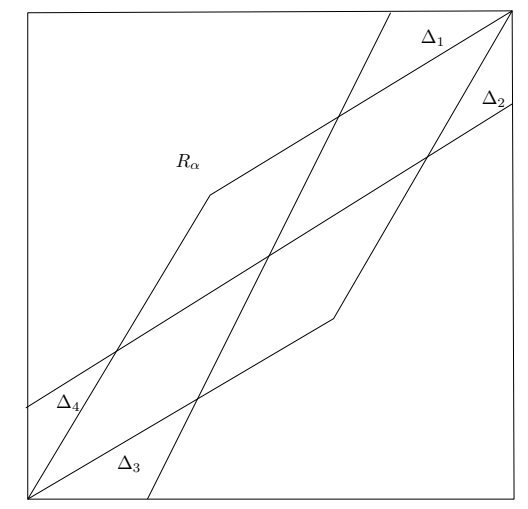

Figure 2: Connecting $I_{m}$ to $R_{\alpha}$.

Claim 3.11. $\mathbb{P}\left(E_{3} \mid E_{1}\right)=1-o(1)$ and thus $\mathbb{P}\left(E_{1} \cap E_{2} \cap E_{3}\right)$ is larger than some $p_{\alpha}^{\prime \prime}$ that does not depend on $n$.

Proof of Claim. By applying Winkler's result and the linear transformation $T^{\alpha}$ we obtain that the points in $R_{\alpha}$ lie in the same component w.h.p.. The event $E_{1}$ implies that all points in $\bigcup_{i=1}^{m} I_{i}$ are contained in the same component and so are those in $\bigcup_{i=1}^{m} J_{i}$. Consider any point $P \in I_{m} \cup J_{m}$. Let $\Delta_{1}, \Delta_{2}, \Delta_{3}, \Delta_{4}$ denote the 4 triangles forming $P^{\|}\left(\frac{1}{2}, \frac{1}{2}\right) \backslash R_{\alpha}$ and let $R_{1}, R_{2}, R_{3}, R_{4}$ denote the 4 parallelograms obtained by dividing $R_{\alpha}$ into 4 equal pieces similar to $R_{\alpha}$ (see Figure 2 ). Observe that, by the definition of $m, P^{\|}$contains one of $\Delta_{i}$ which are of constant size, thus they are non-empty w.h.p., and so are the $R_{i}$ s. If $Q \in \Delta_{i}$, then $Q^{\|}$contains one of the $R_{i} \mathrm{~s}$, thus $P$ is connected to a point of $R_{\alpha}$ by a path of length 2 .

Claim 3.12. If $E_{1}, E_{2}$ and $E_{3}$ hold, then $G_{\alpha, n}^{P}$ is connected.

Proof of Claim. As $E_{2}$ and $E_{3}$ hold, all points lying in $R_{\alpha}$ and $\bigcup_{i=1}^{m}\left(I_{i} \cup J_{i}\right)$ belong to the same component. But $Q \backslash S \subseteq R_{\alpha}^{\|} \cup \bigcup_{i=1}^{m}\left(I_{i}^{\|} \cup J_{i}^{\|}\right)$.

Claim 3.11 and Claim 3.12 finishes the proof of the proposition.

Corollary 3.13. For any fixed $\alpha$ with $0<\alpha<\frac{\pi}{2}$ there exist constants $0<p_{\alpha}, p_{\alpha}^{\prime}<1$ such that

$$
p_{\alpha} \leq \mathbb{P}\left(G_{\alpha, n} \text { is connected }\right) \leq p_{\alpha}^{\prime} .
$$

Proof. As above, the existence of $p_{\alpha}^{\prime}$ follows from the fact that the point $C=\left(\frac{1}{\sqrt{n}}, 1-\right.$ $\left.\frac{1}{\sqrt{n}}\right)$ is a cut point of $G_{\alpha, n}$ with positive probability. For the lower bound, let $X$ be a Poisson variable with mean $(1-\varepsilon) n$ and consider $\mathcal{P}_{\alpha, n}$ as (w.h.p.) $\mathcal{P}_{\alpha,(1-\varepsilon n)}^{P}=\mathcal{P}_{\alpha, X}$ 
with $n-X$ additional points added uniformly at random. The proof of Proposition 3.10 shows that with probability at least $p_{\alpha}, G_{\alpha, X}$ is connected and moreover that any point of $Q \backslash S$ is comparable to some point in $G_{\alpha, X}$. Thus adding points in $Q \backslash S$ will preserve connectivity. But the probability that any additional point lies in $S$ is at most $|S|(n-X)$ which is $\leq \varepsilon$ w.h.p..

Lemma 3.8 tells us that $G_{\alpha, n}$ is disconnected either because of the existence of an isolated vertex or a cutpoint defined by two points in $\mathcal{P}_{\alpha, n}$. To have some more insight on the probability distribution of the number of such points we prove the following two lemmas.

Lemma 3.14. For any fixed $\alpha, 0<\alpha<\frac{\pi}{2}$, let $Y(n, \alpha)$ denote the number of isolated vertices in $G_{\alpha, n}$. Then

$$
\lim _{n \rightarrow \infty} \mathbb{E}(Y(n, \alpha))=\frac{\pi}{2 \tan \alpha} .
$$

Proof. The probability that the vertex corresponding to a point $P_{1}$ is isolated is given by

$$
p:=\int_{0}^{1} \int_{0}^{1}\left(1-\left|P^{\|}(x, y)\right|\right)^{n-1} d x d y .
$$

For large $n$, the square $\left[0, \frac{\log n}{\sqrt{n}}\right] \times\left[1-\frac{\log n}{\sqrt{n}}, 1\right]$ is disjoint from $\left(P^{\|}(0,0) \cup P^{\|}(1,1)\right)$ and thus by Proposition 3.1 for points lying in $\left[0, \frac{\log n}{\sqrt{n}}\right] \times\left[1-\frac{\log n}{\sqrt{n}}, 1\right]$ the area $\left|P^{\|}(x, 1-y)\right|$ is $\left(x^{2}+y^{2}\right) \tan \alpha$ and for points in $\left[1-\frac{\log n}{\sqrt{n}}, 1\right] \times\left[0, \frac{\log n}{\sqrt{n}}\right]$ we have $\left|P^{\|}(1-x, y)\right|=\left(x^{2}+\right.$ $\left.y^{2}\right) \tan \alpha$. For points outside these squares the area $\left|P^{\|}(x, y)\right|$ is at least $\Omega\left(\frac{(\log n)^{2}}{n}\right)$, thus

$$
\begin{aligned}
p & =2 \int_{0}^{\frac{\log n}{\sqrt{n}}} \int_{0}^{\frac{\log n}{\sqrt{n}}}\left(1-\left(x^{2}+y^{2}\right) \tan \alpha\right)^{n-1} d x d y+n^{-\Omega(\log n)} \\
& =2 \int_{0}^{\frac{\log n}{\sqrt{n}}} \int_{0}^{\frac{\log n}{\sqrt{n}}} e^{-n\left(x^{2}+y^{2}\right) \tan \alpha} d x d y+n^{-\Omega(\log n)},
\end{aligned}
$$

where we have used the fact that $e^{-n z}=\left(1-z+O\left(z^{2}\right)\right)^{n}=(1-z)^{n}+O\left(n z^{2}\right)$ for $z<1$, and hence $(1-z)^{n-1}=e^{-n z}+O\left(z+n z^{2}\right)=O\left((\log n)^{4} / n\right)$ when $z=O\left((\log n)^{2} / n\right)$. Writing $u=\sqrt{n \tan \alpha} x, v=\sqrt{n \tan \alpha} y$ we obtain

$$
p=\frac{2}{n \tan \alpha}\left(\int_{0}^{\sqrt{\tan \alpha} \log n} e^{-u^{2}} d u\right)^{2}+n^{-\Omega(\log n)}=\frac{\pi}{2 n \tan \alpha}+n^{-\Omega(\log n)} .
$$

The result now follows since $\mathbb{E}(Y(n, \alpha))=n p$. 
Let us define the following random variables: $Z_{i, j, u, \alpha}\left(Z_{i, j, d, \alpha}\right)$ is the indicator variable of the event that both $P_{i}, P_{j} \in \mathcal{P}_{\alpha, n}$ lie in the half-plane $y \geq x(y \leq x)$, the point $C=e_{\alpha,+, P_{i}} \cap e_{\alpha,-, P_{j}}$ is a cut point of $\mathcal{P}_{\alpha, n}$ and $C$ is closer to the line $x=y$ than $P_{i}$ and $P_{j}$. Note that the events associated with $Z_{i, j, u, \alpha}$ and $Z_{j, i, u, \alpha}$ are not the same. By the result of Winkler [16] and applying the linear transformation $T^{\alpha}$ defined in Section 2, we know that the points of $\mathcal{P}_{\alpha, n}$ lying in the rhombus $R_{\alpha}$ form a connected component. Lemma 3.8 shows that the sum of the random variables $Z_{i, j, u, \alpha}, Z_{i, j, d, \alpha}$ is the number of components that contain more than one vertex and that are different from the component containing the points in $R_{\alpha}$. If we denote the sum by $Z(n, \alpha)$, then we obtain the following proposition.

Proposition 3.15. $G_{\alpha, n}$ is connected if and only if $Y(n, \alpha)+Z(n, \alpha)=0$.

Lemma 3.16. For any $\alpha<\frac{\pi}{2}$ let us write $t_{\alpha}=\tan \left(\frac{\pi}{4}-\frac{\alpha}{2}\right)$. Then

$$
\lim _{n \rightarrow \infty} \mathbb{E}(Z(n, \alpha))=\frac{t_{\alpha}}{\tan \alpha} .
$$

Proof. Let us compute $\mathbb{E}\left(Z_{i, j, u, \alpha}\right)=\mathbb{P}\left(Z_{i, j, u, \alpha}=1\right)$. The intersection point $C=$ $(x, 1-y)$ of $e_{\alpha,+, P_{i}}$ and $e_{\alpha,-, P_{j}}$ determines two line segments on which $P_{i}$ and $P_{j}$ should lie. Allowing the these lines to vary by distances $d u$ and $d v$ perpendicular to themselves causes these lines to trace out two infinite strips $S_{1}$ and $S_{2}$ of widths $d u$ and $d v$ respectively. Now fix these strips and allow $P_{i}, P_{j}$, and $C$ to move. If $P_{i}$ lies in $S_{1}$ and $P_{j}$ lies in $S_{2}$ then $C$ lies in $S_{1} \cap S_{2}$, and conversely, if $C$ lies in $S_{1} \cap S_{2}$ then $P_{i}$ lies in $S_{1}$ and $P_{j}$ lies in $S_{2}$. The intersection of $S_{1}$ and $S_{2}$ is a parallelogram of area $d u d v / \sin \alpha$. Requiring $P_{i}$ to be to the left of $C$ and $P_{j}$ to be above $C$ restricts these points to regions of area approximately $x d v / \cos \left(\frac{\pi}{4}-\frac{\alpha}{2}\right)$ and $y d u / \cos \left(\frac{\pi}{4}-\frac{\alpha}{2}\right)$ respectively. Thus we can estimate the probability that $C$ lies in the parallelogram $S_{1} \cap S_{2}$ given a random choice of $P_{i}$ and $P_{j}$ as $x y d u d v / \cos ^{2}\left(\frac{\pi}{4}-\frac{\alpha}{2}\right)$ and hence the probability density function of the location of $C$ is on average about

$$
\frac{x y d u d v / \cos ^{2}\left(\frac{\pi}{4}-\frac{\alpha}{2}\right)}{d u d v / \sin \alpha}=\frac{x y \sin \alpha}{\cos ^{2}\left(\frac{\pi}{4}-\frac{\alpha}{2}\right)}=2 x y t_{\alpha} \tan \alpha
$$

in a small parallelogram near $(x, 1-y)$. Here we have used the identity $2 t_{\alpha} \cos ^{2}\left(\frac{\pi}{4}-\right.$ $\left.\frac{\alpha}{2}\right)=2 \sin \left(\frac{\pi}{4}-\frac{\alpha}{2}\right) \cos \left(\frac{\pi}{4}-\frac{\alpha}{2}\right)=\sin \left(\frac{\pi}{2}-\alpha\right)=\cos \alpha$ to simplify the trigonometric expression. Thus we have

$$
\mathbb{P}\left(Z_{i, j, u, \alpha}=1\right)=2 t_{\alpha} \tan \alpha \iint_{x+y<1} x y\left(1-\left|C^{\|}(x, 1-y)\right|\right)^{n-2} d x d y .
$$

Using Proposition 3.1 as in the proof of Lemma 3.14, this becomes

$$
2 t_{\alpha} \tan \alpha \int_{0}^{\frac{\log n}{\sqrt{n}}} \int_{0}^{\frac{\log n}{\sqrt{n}}} x y e^{-n\left(x^{2}+y^{2}\right) \tan \alpha} d x d y+n^{-\Omega(\log n)} .
$$


Writing again $u=\sqrt{n \tan \alpha} x, v=\sqrt{n \tan \alpha} y$ we obtain that

$$
\begin{aligned}
\mathbb{E}\left(Z_{i, j, u, \alpha}\right) & =2 t_{\alpha} \tan \alpha\left(\frac{1}{n \tan \alpha} \int_{0}^{\sqrt{\tan \alpha} \log n} u e^{-u^{2}} d u\right)^{2}+n^{-\Omega(\log n)} \\
& =\frac{t_{\alpha}}{2 n^{2} \tan \alpha}+n^{-\Omega(\log n)} .
\end{aligned}
$$

The result follows as $\mathbb{E}(Z(n, \alpha))=n(n-1) \mathbb{E}\left(Z_{i, j, u, \alpha}\right)+n(n-1) \mathbb{E}\left(Z_{i, j, d, \alpha}\right)$.

By Lemma 3.14 and Lemma 3.16 we obtain the following corollary.

Corollary 3.17. If $\mathfrak{P}$ denotes the property of having a connected comparabilty graph, then

$$
\tau(\mathfrak{P})=\frac{\pi}{2} .
$$

There are many examples for hitting-time results in the Erdős-Rényi random graph process that link some monotone graph property to the minimum degree. One of the earliest and most well-known such result is due to Bollobás and Thomason [6]: whenever the minimum degree becomes 1 , the random graph gets connected. We finish this section with a result which shows that the evolution of $G_{\alpha, n}$ and $G_{\alpha, n}^{P}$ is very much different from that of the Erdős-Rényi graph.

Proposition 3.18. For any fixed $\alpha<\frac{\pi}{2}$ there exists a constant $p_{\alpha}^{\prime \prime}>0$ such that

$$
\mathbb{P}\left(G_{\alpha, n}^{P} \text { is disconnected but contains no isolated vertices }\right)>p_{\alpha}^{\prime \prime} .
$$

Proof. We will use the ideas and definitions of Proposition 3.10. Let $E_{2}^{\prime}$ denote the event that $\mathcal{P}_{\alpha, n}^{P}$ does not contain any point in $S^{\prime}=\left(\left[0, x_{2}\right] \times\left[1-x_{1}, 1\right] \cap\left(P^{\|}(0,1-\right.\right.$ $\left.\left.x_{1}\right) \cup P^{\|}\left(x_{2}, 1\right)\right) \cup\left[1-x_{1}, 1\right] \times\left[0, x_{2}\right]$ and contains exactly 2 comparable points in $S^{\prime \prime}=\left(\left[0, x_{2}\right] \times\left[1-x_{1}, 1\right] \backslash\left(P^{\|}\left(0,1-x_{1}\right) \cup P^{\|}\left(x_{2}, 1\right)\right.\right.$. If $E_{1}, E_{3}$ are as defined in Proposition 3.10, then as in the proof of Proposition $3.10 E_{1} \cap E_{2}^{\prime} \cap E_{3}$ implies that $G_{\alpha, n}^{P}$ contains 2 connected components one of which consists of the 2 points of $\mathcal{P}_{\alpha, n}^{P} \cap S^{\prime \prime}$. The events $E_{1}$ and $E_{2}^{\prime}$ are independent as they involve disjoint subsets of $Q$ and their probability is bounded away from 0. Thus we are done by Claim 3.11.

Note that a similar statement for $\mathcal{P}_{\alpha, n}$ holds with a proof identical to that of Corollary 3.13. 


\section{Concluding remarks and open problems}

Theorem 1.1 states that the size of the largest chain in $\mathcal{P}_{\alpha . n}$ is asymptotically $2 \sqrt{n \tan \frac{\alpha}{2}}$ provided $\alpha$ does not tend to $\pi$ or to 0 too quickly. We would be interested to know what can one say when the conditions of Theorem 1.1 are not satisfied. Note that Theorem 3.2 settles the case of extremely quickly convergent $\alpha$ as the proof of its part (i) shows that if $\pi-\alpha=\omega\left(n^{-2}\right)$, then $L_{\alpha, n} \leq n-K$ for any constant $K$. The threshold function of the existence of a chain of length $r$ cannot be deduced directly from Theorem 1.1 (iii), but a very similar proof works of which we give a sketch here.

Theorem 4.1. For any fixed $r \geq 2$ we have

$$
\lim _{n \rightarrow \infty} \mathbb{P}\left(L_{\alpha, n}<r\right)= \begin{cases}1 & \text { if } \alpha=o\left(n^{-\frac{r}{r-1}}\right) \\ 0 & \text { if } \alpha=\omega\left(n^{-\frac{r}{r-1}}\right) .\end{cases}
$$

Proof. W.l.o.g we may assume that $\alpha=o(1)$. Let $X_{i_{1}, \ldots, i_{r}}$ denote the indicator function of the event that $P_{i_{1}} \prec_{\alpha} P_{i_{2}} \prec_{\alpha} \cdots \prec_{\alpha} P_{i_{r}}$ holds. As in the $\alpha$-ordering we have $\left|P^{+}(x, y)\right|=\Theta\left((\min \{1-x, 1-y\})^{2} \alpha\right)$, so we see that $p_{r}:=\mathbb{E}\left(X_{i_{1}, \ldots, i_{r}}\right)=\Theta\left(\alpha^{r-1}\right)$. Thus the expected number of chains of length $r$ is $\mathbb{E}\left(X_{\alpha, r, n}\right)=\Theta\left(n^{r} \alpha^{r-1}\right)$ from which the first statement follows.

Clearly, if $i_{1}, \ldots, i_{r}$ and $i_{1}^{\prime}, \ldots, i_{r}^{\prime}$ are all distinct, then $X_{i_{1}, \ldots, i_{r}}$ and $X_{i_{1}^{\prime}, \ldots, i_{r}^{\prime}}$ are independent and $\mathbb{E}\left(X_{i_{1}, \ldots, i_{r}} X_{i_{1}^{\prime}, \ldots, i_{r}^{\prime}}\right)=p_{r}^{2}$. If $i_{1}, \ldots, i_{r}$ and $i_{1}^{\prime}, \ldots, i_{r}^{\prime}$ have $j \geq 1$ elements in common, then $\mathbb{E}\left(X_{i_{1}^{\prime}, \ldots, i_{r}^{\prime}} \mid X_{i_{1}, \ldots, i_{r}}=1\right)=O\left(\alpha^{r-j}\right)$. Therefore for these pairs of index sequences we have $\mathbb{E}\left(X_{i_{1}, \ldots, i_{r}} X_{i_{1}^{\prime}, \ldots, i_{r}^{\prime}}\right)=O\left(\alpha^{2 r-1-j}\right)$. Summing over all possible $j$ we obtain

$\mathbb{E}\left(X_{\alpha, r, n}^{2}\right)=n_{(2 r)} p_{r}^{2}+\sum_{j=1}^{r} O\left(n^{2 r-j} \alpha^{2 r-1-j}\right)=(1+o(1))\left(n_{(r)} p_{r}\right)^{2}=(1+o(1))\left(\mathbb{E}\left(X_{\alpha, r, n}\right)\right)^{2}$,

where $n_{(r)}=\frac{n !}{(n-r) !}$ and we used $\alpha=\omega\left(n^{-\frac{r}{r-1}}\right)$ for the second equality. The theorem follows from Chebyshev's inequality.

Concerning the connectivity of $G_{\alpha, n}$, the most important open problem is to determine the limiting probability $\lim _{n \rightarrow \infty} \mathbb{P}\left(G_{\alpha, n}\right.$ is connected $)$ for any fixed $0<\alpha<$ $\frac{\pi}{2}$. Another problem that remains open is to calculate the diameter of $G_{\alpha, n}$ when $\alpha=\frac{\pi}{2}-\beta$ with $0<\beta=o(1)$. Note that Corollary 3.17 states that in this case $G_{\alpha, n}$ is connected w.h.p..

Acknowledgement. We would like to thank an anonymous referee for bringing some of the references to our attention. This enabled us to strengthen Theorem 1.1 to its present form. 


\section{References}

[1] D. Aldous, P. Diaconis, Longest increasing subsequences: from patience sorting to the Baik-Deift-Johansson theorem, Bull. Amer. Math. Soc. 36 (1999), 413432 .

[2] J. Baik, P. Deift, K. Johansson, On the distribution of the length of the longest increasing sequence of random permutations, J. Amer. Math. Soc. 12 (1999), 1119-1178.

[3] B. Bollobás, G. Brightwell, The height of a random partial order: concentration of measure, The Annals of Applied Probability 2 (1992), 1009-1018.

[4] B. Bollobás, G. Brightwell, Box spaces and random partial orders, Trans. Amer. Math. Soc. 324 (1991), 347-353.

[5] B. Bollobás, S. Janson, On the Length of the Longest Increasing Subsequence in a Random Permutation, Combinatorics, geometry and probability (Cambridge, 1993), 121-128, Cambridge Univ. Press, Cambridge, 1997.

[6] B. Bollobás, A. Thomason, Random graphs of small order, Random graphs '83 (Poznan, 1983), North-Holland Math. Stud., Vol. 118, North-Holland, Amsetrdam, 1985, pp. 47-97.

[7] B. Bollobás, P. Winkler, The longest chain among random points in Euclidean space, Proc. Amer. Math. Soc. 103 (1988), 347-353.

[8] P. Erdős, A. Rényi, On the evolution of random graphs, Publications of the Mathematical Institute of the Hungarian Academy of Sciences 5, 17-61. (1960)

[9] G. Brightwell, Models of random partial orders, Surveys in combinatorics, 1993 (Keele), 53-83, London Math. Soc. Lecture Note Ser., 187, Cambridge Univ. Press, Cambridge, 1993.

[10] A. Frieze, On the length of the longest monotone subsequence in a random permutation, Ann. Appl. Probab. 1 (1991), 301-305.

[11] J.M. Hammersley, A few seedlings of research, Proceedings of the Sixth Berkeley Symposium on Mathematical Statistics and Probability, 345-394. Univ. California Press, Berkeley, Calif., 1972.

[12] J.H. Kim, On increasing subsequences of random permutations, J. Combin. Theory Ser. A 76 (1996), 148-155. 
[13] B.F. Logan, L.A. Shepp, A variational problem for random Young tableaux, Advances in Math. 26 (1977), 206-222.

[14] S.M. Ulam, Monte Carlo calculations in problems of mathematical physics, Modern mathematics for the engineer: Second series, pp. 261-281 McGraw-Hill, New York, 1961.

[15] A.M. Vershik, S.V. Kerov Asymptotic behavior of the Plancherel measure of the symmetric group and the limit form of Young tableaux. (Russian) Dokl. Akad. Nauk SSSR 233 (1977), 1024-1027.

[16] P. Winkler, Random orders, Order 1 (1985), 317-335.

[17] P. Winkler, Connectedness and diameter for random orders of fixed dimension, Order 2 (1985), 165-171. 